\title{
A SURVEY ON MAGNETIC WALL-CLIMBING ROBOTS FOR INSPECTION
}

\section{JAISE JOSE, D. DINAKARAN, M. M. RAMYA \& D. G. HARRIS SAMUEL}

Centre for Automation \& Robotics, Hindustan Institute of Technology and Science, Chennai, Tamil Nadu, India

Ferromagnetic surfaces in the industry such as Gas and oil tanks require periodical inspections for detection of cracks, corrosion, material degradation and welding defects. The workers carrying on these operations have to face hazardous environments and this process is time-consuming. A climbing robot would ease these processes by eliminating the risks of the employees and will improve the inspection time considerably. Thus, the magnetic adhesion remains a growing application area of research in mobile robots for non-destructive testing of various surfaces. Design of such robots requires equipping them with proper locomotion, adhesion mechanisms and the right adaptation for carrying the appropriate tools and sensors. This paper presents the survey of the magnetic systems for the wall climbing robots for inspection with the comprehensive detailing of classification, locomotion and its applications.
\end{abstract}

KEYWORDS: Wall Climbing Robot, Magnetic Adhesion, Inspection \& Non-Destructive Testing

Received: Aug 31, 2018; Accepted: Aug 21, 2018; Published: Oct 15, 2018; Paper Id.: IJMPERDDEC20186

\section{INTRODUCTION}

Often, the inspectors have to climb up, hang on cables, or have to use special kind of lifters to inspect large bridges, ship hulls and other industrial infrastructures with high attitudes (figure 1). Some areas of the complex industrial structures are hard to reach or may not be accessible due to their confined space and other complexities. These problems can be solved by using climbing systems or the climbing robots. The robotic systems for moving over variety of environments such as walls, ceilings, roofs, and other high altitude structures and for conducting various tasks such as welding inspection maintenance and so on is classified under Climbing robots.

The Ferro magnetic surface climbing has been the field of interest lately with the inspection tasks being automated and the concerns over the security of human lives. Therefore, climbing robots are seen as a great alternatives with great potential, which would enhance the efficiency of operation several times and decrease or could possibly eliminate the hazardous tasks involved in the inspection and evaluation of storage tanks in the Petrochemical facilities, oil and storage tanks, nuclear storage tanks, welding and maintenance of ship hulls, and so on which are mainly made up of Ferro magnetic material.

The wall climbing robots dates back to the 1960s, (Nishi et al, 1986) was able to climb over the vertical walls and the ceilings but was large in size and bulky. And ever since this, the wall climbing robots are being built for various purposes using various technologies. The most common adhesion methods (Maempel et al, 2010) used by the Wall climbing robots can be broadly classified into either magnetic, pneumatic, gripping type the Biometric or the biologically inspired types. 

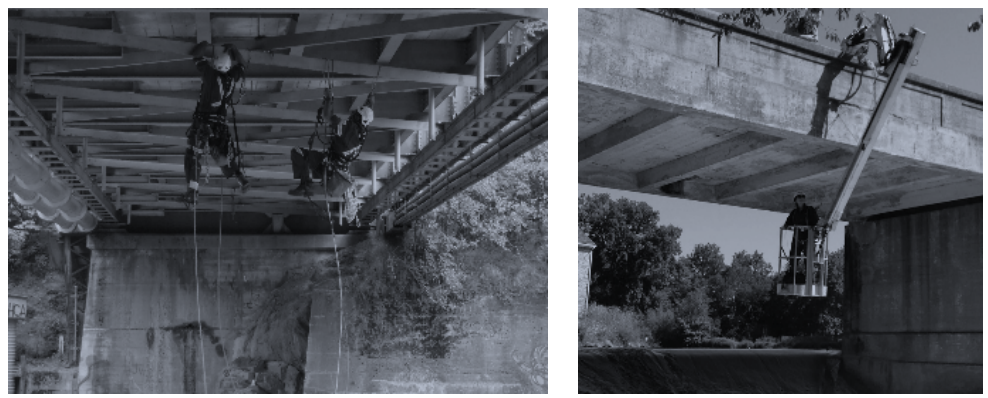

(Sources: 'Harcon Corporation' Website, 'Working AT Height Alti-Service’ Website)

Figure 1: Inspection Tasks Performed by Inspectors

Here, the focus is on the magnetic system of adhesion in mobile robots, and its applications specifically for the non-destructive techniques for inspection.

Adhesion and locomotion combinations with variety of configurations (Chu Nishi et al, 2010)(Daniel et al, 2013), (Brusell et al, 2016), (Armada et al, 2003) are used to solve the problems of the Wall-Climbing Robots according to the requirement, while remaining adhered onto the surfaces of varying inclinations.

\section{ADHESION PRINCIPLES OF MAGNETIC ADHESION}

Adhesion mechanism, the magnetic adhesion falls under two categories (Wang, Q., 2013) namely, permanent magnet and electro magnet adhesion mechanisms

\section{Permanent Magnetic Adhesion}

Permanent magnet creates its own persistent magnetic field, and this type of adhesion units does not require external power sources to maintain adhesion and have strong adsorption capacity between the permanent magnet, and the adsorbed surface the permanent magnets are used in various configurations in the mobile robots. The variation of magnetic flux is achieved using the Switchable magnets or some other mechanism, by which the distance can be varied between the magnets and the surface

Permanent magnetic adhesion deploys mainly $\mathrm{NdFeB}$, as it has high coercivity, rich resources energy product, and cheap, thus is the most widely used.

\section{Electro-Magnetic Adhesion}

In electro magnets, the magnetic field is produced by an electric current passing through coil, and when the current is turned off, the magnetic field gets to zero. The adhesion of electromagnet can be varied according to the current flowing through the coil. However, this electromagnet requires constant supply of power unlike the permanent magnet that needs no power. This adhesion mechanism is specifically useful where, the variable adhesion is required and the speed of switching required is high, and the power supply constraints are not there.

\section{LOCOMOTION}

The adhesion is configured according to the surfaces and the planned locomotion, and major classifications are based on locomotion namely a) Legged Locomotion b) Wheel-driven motion and c) Tracked Locomotion 


\section{Legged Locomotion}

One of the common mechanisms is the adoption of legs of the living beings. Magnetic climbing robots employ around four up to eight legs. To stay and climb the walls, the magnetic adhesion is deployed at the end of the foot of the robot. This kind of mechanism enables movement over rough surfaces, cracks, and other obstacles while having stable adherence to the surface. The number of legs will increase the stability of the robot and improve the adaptability and the adherence to the wall. However; the increase of legs will be resulting in complex control system as the system will use harmonic gait control (García-López et al, 2012). Apart from this, the major disadvantage of discontinuous movement is the relatively low speed of operation. The implementation of legged motion is implemented in different ways in various robots.

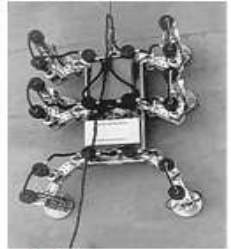

(1.1)

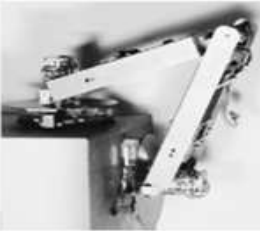

(1.2)

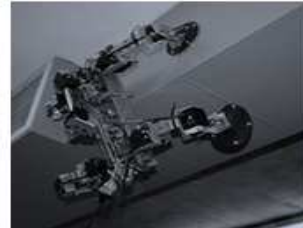

(1.3)

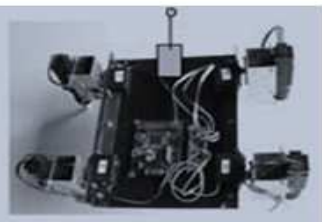

(1.4)

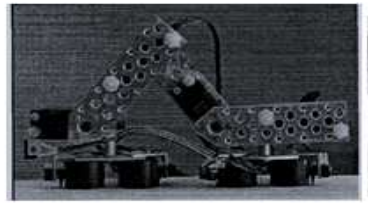

(1.5)

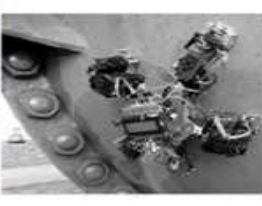

(1.6)

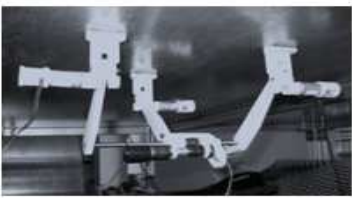

(1.7)

Figure 2: Legged Locomotion:

(1. 1) Rest 1(J. Grieco et al, 1998), (1. 2) Tremo (F. Rochatet et al, 2011),

(1. 3) Reconfigurable Vertical Climber (RVC) (G. Peters et al, 2010),

(1. 4) Inchworm (K. Kotay at al, 1996) (1. 5) 4Steel (De Los, et al, 2007), (1. 6) Winspecbot (B. Kamagaluh et al, 2012), (1. 7) Mag-Foot

(Mazumdar. A and H. H. Asada, 2009)

The climbing robots, which use the legged locomotion for the movement (Grieco et al, 1998), (K. Kotay et al, 1996) the end of legs are fitted with electromagnet based grasping devices, enabling the robot to adhere to the ferromagnetic-material walls with safely. The use of electromagnets help in reducing the weight and the placement of power supply outside the robot body help reducing the bulk of robot, however, the trade-off is the energy supply required cannot be tuned off, and it consumes more power

The legged robots explained in (F. Rochat et al, 2012), (De Los et al, 2007), (M. Eich et al, 2012), (Mazumdar et al, 2009) use permanent magnetic adhesion and they deploy magnetic pad at the end-effectors of each leg, and for better adhesion, each robot has unique way of placing magnets and varying the magnetic field strength of these magnets for detachment from the surface. Unlike the electromagnets, the magnetism of permanent magnet cannot be varied, so various methods are adopted in the above mentioned robots to vary the magnetic strength of the magnets. The air gap adjustment using a servomotor, Magnetic switchable devices (MSD), Tilting foot mechanism are some methods which are used for the purpose. This kind of switching has better efficiency compared to the electromagnetic detachment. In this type of locomotion, the legs have to detach and in various circumstances, thus the center of gravity may be dislocated, thus the stability may be affected and so proper care has to be taken for be re-orientation of the gravity center using control mechanisms and the adhesion mechanisms of the robot. 


\section{Wheeled Locomotion}

Wheeled locomotion or Wheel-driven locomotion facilitates continuous magnetic adhesion force application that allows for their unceasing motion. This motion provides good speed improvement, but at the cost of weak obstacle, avoidance and slipping, if proper care not taken of. Different wheel configurations are used for locomotion like Omnidirectional wheels, two independently controlled wheels, conventional four wheel configurations and more. Adhesion required is provided by magnetic wheels, wheels embedded with magnets, magnetic roller or separate magnets placed close to the surface.

The motion and direction change of the robot on the surface is achieved by using various methods to achieve target motion for the purpose. The (J. Sanchez et al, 2006) uses two DC motors, one motor produces motion and the other motor decides the change in direction. The belt drive connects the motors and the wheels. Places which require high maneuverability and flexibility, the robots (M. Tavakoli et al, 2012) (J. Li and X. Song Wang, 2016) with Omni wheels (Tang et al, 2012) are used for locomotion. With its unique design is provided for movement in any (x, y) coordinate and rotate around it. These kinds of wheels are either applied in three or four wheel configuration in the mobile robots. These kinds of wheels provide maximum normal force to the surface attached, and allow the robot to be more independent of the direction of gravity, and thus getting better adhesion to the surface.

Air Gap Crawler (W. Fischer et al, 2009) uses a special kind of arrangement for very thin design of robots (8mm) that can travel between the rotor and stator of electrical generators for inspection. Flexible magnetic roller wheel pairs are used with a spur gear transmission for locomotion. The MagneBike (F. Tâche et al, 2007) uses a bicycle like configuration, with 2 distinct magnetic wheels enhanced with lifter. These lifter help varying the magnetic strength at curved edges, also it helps stabilizing the wheels and navigates through complex industrial structures (Schoeneich et al, 2012). A modular design is used with four main modules, and each module is fitted with magnetic wheels and the locomotion module has a motor, driving a magnetic wheel which drives the entire robot in the environments, which requires the body of the robot to be highly flexible. Robot (Rui Wang et al, 2012) (W. Fischer et al, 2007) uses four wheels for locomotion and for adhesion, and for changing the direction of motion differential steering is used, whereas the robots specified (Shang et al, 2012), (M. Eich et al, 2012) are driven by two wheels and use additional support wheels for the supporting the robot for stability. 


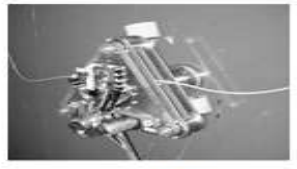

(3.1)

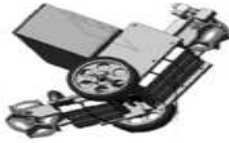

(3.2)

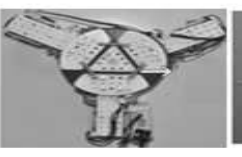

(3.3)

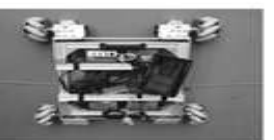

(3.4)

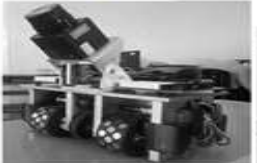

(3.5)

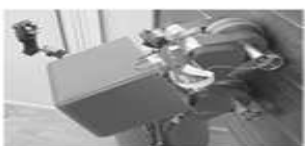

(3.6)

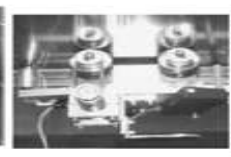

(3.7)

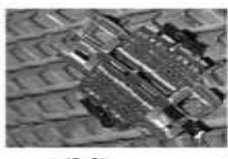

(3.8)

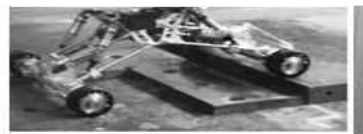

(3.8)

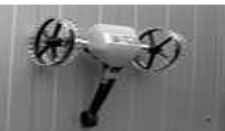

(3.9)

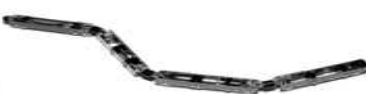

(3.10)

Figure 3: Wheeled Locomotion:

(3. 1) Robot (J. Sanchez et al, 2006), (3. 2) NDT Robot [19]

(3. 3) Omni climber (M. Tavakoli et al, 2012) (3. 4) Robot

(J. Li and X. Song Wang, 2016) (35) Robot(Shang et al, 2012),

(3.6) The MagneBike (F. Tâche et al, 2007) (3.7) Robot [22]

(3.8) AirGapCrawler (W. Fischer et al, 2009) [33], (3.9)

MINOAS ( M. Eich and T. Vögele, 2011) (6.10)

TubeCrawler (Schoeneich et al, 2012)

\section{Tracked Locomotion}

Tracked locomotion also uses rotational mechanism in a different way. A chain-track is used, wherein two frames that can move in relation to one other according to the direction of the motion required. Adhesion is achieved using a number of magnets, used on the chain-track for adhering to the surface, and in alternating steps each frame attaches to the target surface as the other frame moves into its new position. This tracked locomotion is comparable to the motion of the caterpillars. Implementation of tracked locomotion varies in each design according to the requirements. These tracked motions can avoid obstacles efficiently, and provide a large contact area which enables strong adhering to the surface.

For the locomotion (WeiminShen, J. Gu and YanjunShen, 2005) (H. Schempf et al, 1995) (ang et al, 2015) (ang et al, 2015) (Rochat et al, 2011) magnetic tracks driven by motors are used. The adhesive force required for adhering to the surface is supplied by the magnetic track, which is either composed of magnetic material, use separate magnets for adhesion or has slots to accommodate the magnets. The adhesive force needs to be varying in some cases, and the permanent magnets used are switchable according to the motion pattern required. Two motors are utilized for the locomotion. One is employed to driving two roller-chains, and another motor is used to drive the transformation of the robot to approach different contour surfaces such as steel bridges. 


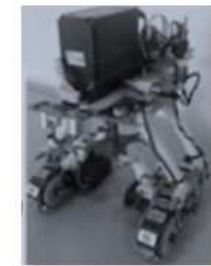

$(4.1)$

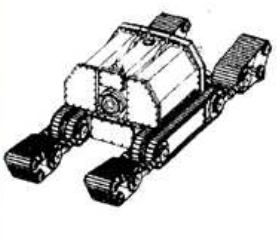

(4.2)

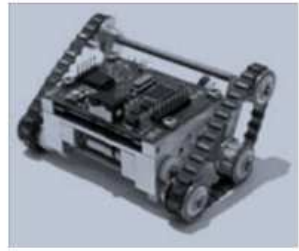

(4.3)

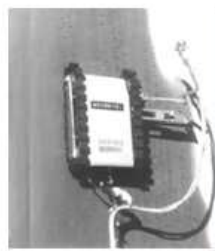

(4.5)

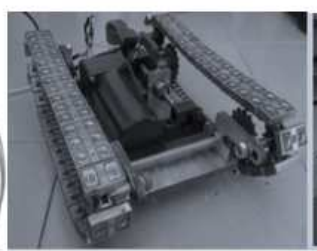

(4.6)

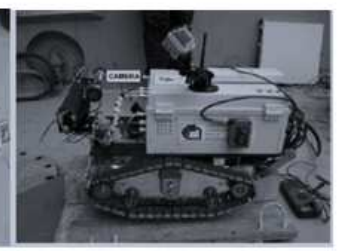

(4.7)

Figure 4: (4. 1) Robot [36] (4. 2) Neptune [34] (4. 3) Tripillar [34]

(4. 4) Robot [38] (4. 5) robot [37] (4. 6) MARC (Maglietta, $R$ et al, 2018)

\section{INSPECTION APPLICATIONS OF MAGNETIC WALL-CLIMBING ROBOT}

Magnetic Wall, climbing Robotic systems find a wide range of applications on target surfaces such as Shipbuilding components, pipelines, oil tanks containers, storage tanks, steam chests, Boiler tubes, and steel bridges and so on, as most of these materials are Ferro-magnetic.

Inspection of Industrial Infrastructures like ship hulls, surface of the storage oil containers and so on, are hazardous and tedious processes conducted by inspectors. The operation involves in either high altitudes or the chemical environments or both. The robot would need to be able to navigate hard to navigate places and accomplish remotely the ultrasonic and visual inspections in large infrastructures (S. R. Armando et al, 2007) (J. Griecoet et al, 1998) are used for inspecting oil tanks and containers. Inspection of Shipbuilding/maintenance with all new concerns about ocean safety (Szlapczynski, R. and Szlapczynska, J., 2017.) inspection operations have become complex and hectic processes for a human inspector. The processes include, weld line monitoring and other high altitude operations in and monitoring of ship hulls, which include areas not easily accessible to humans. As the major components of the ship are made up of Ferromagnetic material, they make the case for the magnetic wall climbing robots. The REST 1 (Grieco, J. C. et al, 1998) NDT robot (Shang et al, 2012), Modular Climbing Robot, MARC (Maglietta, R et al, 2018), The MINOAS (M. Eich et al, 2012) are some of the wall climbing robots, which ease these operations in this industry.

In the power generation sector, variety of hard to be inspected components like boiler tubes, steam chests and the stator or rotor of generators can be inspected using robots. Boiler tubes are generally ferromagnetic tubes, which are around $25 \mathrm{~mm}$ in diameter and may be situated in any in any inclination. And, any failure would cost millions, so its inspection is critical to the safety of operation. With the help of the flexible modular body design Tube Crawler (P. Schoeneich et al, 2012) enables the Boiler tubes to be inspected to prevent failure during operations. The stator and rotor in Power plant generators is another area, which requires regular monitoring. This inspection is critical to assure the plant works are safe and reliable. Air Gap Crawler (W. Fischer et al, 2009) miniature robot, with a set of magnetic wheels is in a position to monitor the air gap $(8 \mathrm{~mm})$ between the rotor and stator. Coal and nuclear power plants require regular inspections in the piping system for detecting any possible defects. For inspection, human inspectors have their limitation, as the environments are harsh and have limited accessibility to the site. Robots like Magne Bike (F. Tâche et al, 2009) helps in the inspecting processes of inner casing of such ferromagnetic pipes and steam chests. These robots are integrated 
with all the electronics to handle these complex-shaped structures.

The inspection of civil infrastructure like steel bridge and the base of wind turbines need to be checked for the delamination and corrosion on a regular basis. The inspectors have to climb, use lifters or hang on cables to inspect large bridge and high infrastructures (A McCrea et al, 2002) (Mazumdar et al, 2009). And, as the material is ferromagnetic, it can be accomplished by using the magnetic climbing robots (ang et al, 2015) is capable of climbing on different steel bridge to perform inspection while strongly adhering to the surface of different complexities.

\section{CONCLUSIONS}

This paper presented the survey of Magnetic wall climbing robots, which could be used for nondestructive testing of ferromagnetic surfaces briefly. Different principles for robot locomotion and adhesion were reviewed, and each of its pros and cons were discussed. The paper also looked into its various applications in industries, where these kinds of robots were used.

As a conclusion to this, it is certain that with automation invading the industry, the monitoring and inspection systems needs improvement, especially in unmanned operations with improved efficiency, speed and precision. Thus, wall climbing robots operations remains an important field of research in keeping the infrastructure healthy and functioning.

\section{REFERENCES}

1. Armada, M., De Santos, P. G., Jiménez, M. A. and Prieto, M., 2003. Application of CLAWAR machines. The International Journal of Robotics Research, 22(3-4), pp.251-264.

2. Brusell, A., Andrikopoulos, G. and Nikolakopoulos, G., 2016, June. A survey on pneumatic wall-climbing robots for inspection. In Control and Automation (MED), 2016 24th Mediterranean Conference on (pp. 220-225). IEEE.

3. Chu, B., Jung, K., Han, C. S. and Hong, D., 2010. A survey of climbing robots: Locomotion and adhesion. International journal of precision engineering and manufacturing, 11(4), pp.633-647.

4. De Los, R. A. S., Garduño, G. M. and González, L. A., 2007, October. 4steel-robot: a climbing mobile robot for gas containers inspection. In Proceedings of the 3rd WSEAS/IASME international conference on Dynamical systems and control (pp. 200205). World Scientific and Engineering Academy and Society (WSEAS).

5. Eich, M. and Vögele, T., 2011, June. Design and control of a lightweight magnetic climbing robot for vessel inspection. In Control \& Automation (MED), 2011 19th Mediterranean Conference on (pp. 1200-1205). IEEE.

6. Fischer, W., Caprari, G., Siegwart, R. and Moser, R., 2009, April. Robotic crawler for inspecting generators with very narrow air gaps. In Mechatronics, 2009. ICM 2009. IEEE International Conference on (pp. 1-5). IEEE.

7. Fischer, W., Tâche, F. and Siegwart, R., 2007. Inspection system for very thin and fragile surfaces, based on a pair of wall climbing robots with magnetic wheels. In 2007 IEEE/RSJ International Conference on Intelligent Robots and Systems: San Diego, CA, 29 October-2 November 2007 (pp. 1216-1221). IEEE.

8. García-López, M. C., Gorrostieta-Hurtado, E., Vargas-Soto, E., Ramos-Arreguín, J. M., Sotomayor-Olmedo, A. and Morales, J. M., 2012. Kinematic analysis for trajectory generation in one leg of a hexapod robot. Procedia Technology, 3, pp.342-350.

9. Grieco, J. C., Prieto, M., Armada, M. and De Santos, P. G., 1998, September. A six-legged climbing robot for high payloads. In Control Applications, 1998. Proceedings of the 1998 IEEE International Conference on (Vol. 1, pp. 446-450). IEEE.

10. 'Harcon Corporation' website, https://www.harconcorp.com/bridge-trackers.asp 
11. Kotay, K. D. and Rus, D. L., 1996, November. Navigating $3 d$ steel web structures with an inchworm robot. In Intelligent Robots and Systems' 96, IROS 96, Proceedings of the 1996 IEEE/RSJ International Conference on (Vol. 1, pp. 368-375). IEEE.

12. Kamagaluh, B., Kumar, J. S. and Virk, G. S., 2012. DESIGN OF MULTI-TERRAIN CLIMBING ROBOT FOR PETROCHEMICAL APPLICATIONS. In Adaptive Mobile Robotics (pp. 639-646).

13. Li, J. and Wang, X. S., 2016, November. Novel omnidirectional climbing robot with adjustable magnetic adsorption mechanism. In Mechatronics and Machine Vision in Practice (M2VIP), 2016 23rd International Conference on (pp. 1-5). IEEE.

14. Mazumdar, A. and Asada, H. H., 2009, October. Mag-foot: A steel bridge inspection robot. In Intelligent Robots and Systems, 2009. IROS 2009. IEEE/RSJ International Conference on (pp. 1691-1696). IEEE.

15. Maempel, J., Koehring, S., Schilling, C. and Witte, H., 2010, June. Using different adhesion technologies in modular robot for climbing. In Robotics (ISR), 2010 41st International Symposium on and 2010 6th German Conference on Robotics (ROBOTIK) (pp. 1-7). VDE.

16. McCrea, A., Chamberlain, D. and Navon, R., 2002. Automated inspection and restoration of steel bridges-a critical review of methods and enabling technologies. Automation in Construction, 11(4), pp.351-373.

17. Nishi, A., Wakasugi, Y. and Watanabe, K., 1986. Design of a robot capable of moving on a vertical wall. Advanced Robotics, 1(1), pp.33-45.

18. Pham, N. H. and La, H. M., 2016, September. Design and implementation of an autonomous robot for steel bridge inspection. In Communication, Control, and Computing (Allerton), 2016 54th Annual Allerton Conference on (pp. 556-562). IEEE.

19. Rochat, F., Beira, R., Bleuler, H. and Mondada, F., 2012. Tremo: an inspection climbing inchworm based on magnetic switchable device. In Field Robotics (pp. 421-428).

20. Rochat, Frederic \& Schoeneich, Patrick \& Truong-Dat Nguyen, Olivier \&Mondada, Francesco \&Tosun, $O \& L$. Akin, H \&Tokhi, Mohammad \& Virk, Gurvinder. (2011). TRIPILLAR: Miniature magnetic caterpillar climbing robot with plane transition ability. Robotica. 29. 10.1017/S0263574711000257.

21. Schmidt, D. and Berns, K., 2013. Climbing robots for maintenance and inspections of vertical structures-A survey of design aspects and technologies. Robotics and Autonomous Systems, 61(12), pp.1288-1305.

22. Schoeneich, P., Rochat, F., Nguyen, O. T. D., Caprari, G., Moser, R., Bleuler, H. and Mondada, F., 2010, October. Tubulo-A train-like miniature inspection climbing robot for ferromagnetic tubes. In Applied Robotics for the Power Industry (CARPI), 2010 1st International Conference on (pp. 1-5). IEEE.

23. Shang, J., Bridge, B., Sattar, T., Mondal, S. and Brenner, A., 2008. Development of a climbing robot for inspection of long weld lines. Industrial Robot: An International Journal, 35(3), pp.217-223.

24. Sánchez, J., Vázquez, F. and Paz, E., 2006. Machine vision guidance system for a modular climbing robot used in shipbuilding. In Climbing and Walking Robots (pp. 893-900). Springer, Berlin, Heidelberg.

25. Tavakoli, M., Marques, L. and de Almeida, A. T., 2012, October. Omniclimber: An omnidirectional light weight climbing robot with flexibility to adapt to non-flat surfaces. In Intelligent Robots and Systems (IROS), 2012 IEEE/RSJ International Conference on (pp. 280-285). IEEE. 
26. Tâche, F., Fischer, W., Siegwart, R., Moser, R. and Mondada, F., 2007, October. Compact magnetic wheeled robot with high mobility for inspecting complex shaped pipe structures. In Intelligent Robots and Systems, 2007. IROS 2007. IEEE/RSJ International Conference on (pp. 261-266). IEEE.

27. Tang, X., Zhang, D., Li, Z. and Chen, J., 2012. An omni-directional wall-climbing microrobot with magnetic wheels directly integrated with electromagnetic micromotors. International Journal of Advanced Robotic Systems, 9(1), p.16.

28. Wang, R. and Kawamura, Y., 2014, June. A magnetic climbing robot for steel bridge inspection. In Intelligent Control and Automation (WCICA), 2014 11th World Congress on (pp. 3303-3308). IEEE.

29. Wani, S. F., Ashish, D. K., Dar, M. A., \& Kumar, R. A. V. I. (2015). Study on mix design \& hardened properties of selfcompacting concrete. Int. J. Civil, Struct. Envir. Infrastruct. Eng. Res. Develop, 5(4), 1-10.

30. Wang, Q., 2013. Practical design of magnetostatic structure using numerical simulation. John Wiley \& Sons.

31. WeiminShen, J. Gu and YanjunShen, "Permanent magnetic system design for the wall-climbing robot," IEEE International Conference Mechatronics and Automation, 2005, pp. 2078-2083 Vol. 4.

32. 'Working AT Height Alti-Service' website http://workingatheight.ie/bridge-inspections. 
